\title{
Systemic Immune-inflammation Index is the Best Prognostic Factor in Patients with Advanced Stage Adenocarcinoma of the Lung Treated with Pemetrexed
}

\author{
Irem Bilgetekin and Fatma Bugdayci Basal \\ Department of Medical Oncology, HSU Dr. Abdurrahman Yurtaslan, Oncology Training and Research Hospital, Ankara, Turkey
}

\begin{abstract}
Objective: To evaluate the prognostic role of systemic immune-inflammation index (SII), neutrophil-to-lymphocyte ratio (NLR), and platelet-to-lymphocyte ratio (PLR) in patients who received platin-pemetrexed combination therapy and/or maintenance pemetrexed therapy.

Study Design: Observational study.

Place and Duration of Study: Department of Medical Oncology, HSU Dr. Abdurrahman Yurtaslan Oncology, Training and Research Hospital, Turkey, between January 2010 and March 2020.

Methodology: Data of patients with metastatic adenocarcinoma of lung, who underwent platin-pemetrexed combination therapy and/or maintenance pemetrexed therapy retrospectively, were evaluated. Patient characteristics and disease parameters were recorded. Moreover, NLR, PLR, and SII were calculated. Survival analysis with the Kaplan-Meier and Log-rank test was performed. Cox regression analysis was used to determine independent prognostic factors of overall survivall (OS) and progression-free survival (PFS).

Results: In the univariate analyses, NLR-low group and SII-low group had significantly longer PFS compared to NLR-high and SII-high groups ( 10 months vs. 8 months, $p=0.018$, and 13 months vs. 8 months, $p<0.001$, respectively). The significant differences were seen between SII-low and SII-high groups for OS (24 months vs. 13 months, $p=0.001$ ). In multivariate analyses, response to treatment and low-SII were independent prognostic factors for PFS (HR: $0.25, p<0.001$, and HR: $0.47, p=0.002$, respectively) and OS (HR: 2.09, $p=0.001$, and HR: 2.05, $p=0.001$, respectively).

Conclusion: SIl is the most powerful of the three studied inflammatory indices, which could independently predict overall and progression-free survival.
\end{abstract}

Key Words: Systemic immune-inflammation index, Neutrophil-to-lymphocyte ratio, Platelet-to-lymphocyte ratio, Adenocarcinoma, Lung cancer, Pemetrexed.

How to cite this article: Bilgetekin I, Basal FB. Systemic Immune-inflammation Index is the Best Prognostic Factor in Patients with Advanced Stage Adenocarcinoma of the Lung Treated with Pemetrexed. J Coll Physicians Surg Pak 2020; 30(09):933-939.

\section{INTRODUCTION}

Non-small cell lung cancer represents about $80-85 \%$ of all newly diagnosed lung cancer cases; and the most common type of non-small cell lung cancer is adenocarcinoma. ${ }^{1,2}$ Approximately $75 \%$ of patients with NSCLC are in advanced stage at the time of diagnosis. ${ }^{3}$ Inflammation is considered as a promoting factor in tumorigenesis and progression. ${ }^{4}$ Inflammatory reaction in tumor development and progression is complex and includes many immune cell types as well as cytokines and chemokines. Inflammation has a dual role in tumorigenesis.

Correspondence to: Dr. Irem Bilgetekin, Department of Medical Oncology, HSU Dr. Abdurrahman Yurtaslan, Oncology Training and Research Hospital, Ankara, Turkey E-mail:irembilgetekin@gmail.com

Received: July 14, 2020; Revised: September 04, 2020;

Accepted: September 17, 2020

DOI: https://doi.org/10.29271/jcpsp.2020.09.933
Inflammation is both cause and effect in tumor growth and progression. ${ }^{5}$ Systemic inflammatory markers, to some extent, can reflect local tumor microenvironment, which involves innate immune cells, including macrophages, neutrophils, and lymphocytes, among many other cell types. Thus, careful examination of peripheral blood immune cells and cytokines might provide valuable information with respect to local tumor growth. ${ }^{5,6}$

Clinical studies demonstrated the importance of inflammatory response in tumor invasion, progression and metastasis by regenerating inflammation for angiogenesis and reduced anticancer activity. ${ }^{7}$ Several immune-inflammatory-based prognostic tools, such as neutrophil-to-lymphocyte ratio (NLR), and platelet-to-lymphocyte ratio (PLR) showed the prediction value of recurrence and survival in malignant tumors. ${ }^{8}$ Recent years witnessed the ever-increasing use of these inflammatory scores and indices to predict prognosis in a broad range of disorders. Particularly, NLR has enjoyed a great popularity among 
inflammatory indices, thanks to its availability and strong association with prognosis in a series of cancers. ${ }^{9,10}$ Neutrophils provide tumor adhesion by secreting chemokines and cytokines to facilitate distant metastasis. ${ }^{7}$ Platelets protect circulating tumor cells from immune attack of the system and develop transendothelial migration; while lymphocytes suppress tumor cell proliferation and migration. ${ }^{7,8}$ In this sense, a novel systemic immune-inflammation index (SII), based on platelet, neutrophil and lymphocyte counts, might show the ability of tumor angiogenesis, adhesion, metastasis and immune clearance of cancer cells. ${ }^{7}$ Previous studies focused on inflammatory markers such as NLR, and PLR in NSCLC patients. Compared to these markers, SII may be a better prognostic marker to represent the relationship between host inflammatory and immune status. ${ }^{8}$

The current study aimed to assess inflammatory markers including SII in patients with lung adenocarcinoma treated with platin-pemetrexed combination and/or maintenance pemetrexed.

\section{METHODOLOGY}

This was a retrospective analysis of patients with metastatic lung adenocarcinoma who had been administered platin-pemetrexed as first-line treatment at Oncology Clinic, HSU Dr Abdurrahman Yurtaslan Oncology Training and Research Hospital, Turkey, between January 2010 and March 2020.

The patients who had been administered at least 6 cycles of platin-pemetrexed combination as first-line treatment and/or maintenance treatment of pemetrexed formetastaticlung adenocarcinoma were included in the study. Exclusion criteria were: having non-metastatic lung adenocarcinoma, histologic subtypes of lung cancer other than adenocarcinoma, administering a different first-line treatment in metastatic stage, having any molecular mutations such as EGFR, ALK, and ROS1.

Demographic and clinical data of study participants were retrospectively collected from patient's charts and hospital's electronic database system. Chest and abdominal tomography were used to evaluate the response to treatment. In addition, patients with neurological symptoms had brain MR. Response of treatment was evaluated by using RECIST criteria 1.1. ${ }^{11}$

Several indices were calculated that have been shown to reflect the inflammatory and immune status of the patients. These parameters were assessed one week before the start of combination chemotherapy and one week after sixth cycle of chemotherpy, i.e before the given maintenance dose in patients. Neutrophil to lymphocyte ratio was calculated as peripheral blood absolute neutrophil count divided by absolute lymphocyte count. In a similar way, platelet to lymphocyte count was calculated as platelet count divided by absolute lymphocyte count. Systemic immune-inflammatory index was calculated with the following equation: (Absolute neutrophil count x platelet count) / absolute lymphocyte count. The absolute cut-off value of SII, NLR, and PLR in predicting OS was analysed by the receiver operating characteristics (ROC) curve anal- ysis. The categorisations were generated due to the cut-off values of the parameters.

The overall survival (OS) was described as the time from the start of the combination treatment until death or last follow-up. The progression-free survival (PFS) was described as the time from the start of the combination treatment to disease progression or death.

Table I: General clinical and demographic features of the patients.

\begin{tabular}{|c|c|c|}
\hline \multicolumn{2}{|l|}{ Features } & $\mathrm{N}(\%)$ \\
\hline \multicolumn{3}{|l|}{ Age } \\
\hline & $<65$ & $84(68.3)$ \\
\hline & $\geq 65$ & $39(31.7)$ \\
\hline \multicolumn{3}{|l|}{ Gender } \\
\hline & Male & $96(78.0)$ \\
\hline & Female & $27(22.0)$ \\
\hline \multicolumn{3}{|c|}{ Smoking at diagnosis } \\
\hline & Never & $24(19.5)$ \\
\hline & Current or ex-smoker & $99(80.5)$ \\
\hline \multicolumn{3}{|c|}{ Metastatic disease at diagnosis } \\
\hline & No & $18(14.6)$ \\
\hline & Yes & $105(85.4)$ \\
\hline \multicolumn{2}{|c|}{ Liver metastasis, yes } & $31(25.2)$ \\
\hline \multicolumn{2}{|c|}{ Contralateral lung metastasis, yes } & $49(39.8)$ \\
\hline \multicolumn{2}{|c|}{ Lymph node metastasis yes } & $82(66.7)$ \\
\hline \multicolumn{2}{|c|}{ Bone metastasis, yes } & $64(52.0)$ \\
\hline \multicolumn{2}{|c|}{ Brain metastasis, yes } & $44(35.8)$ \\
\hline \multicolumn{3}{|c|}{ Radiotherapy } \\
\hline & No & $55(44.7)$ \\
\hline & Yes & $68(55.3)$ \\
\hline \multicolumn{3}{|c|}{ Response } \\
\hline & Complete response & $0(0.0)$ \\
\hline & Partial response & $55(44.7)$ \\
\hline & Stable disease & $45(36.6)$ \\
\hline & Progressive disease & $23(18.7)$ \\
\hline \multicolumn{3}{|c|}{ 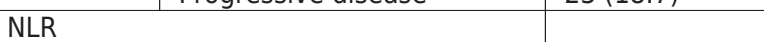 } \\
\hline & $<3.3$ & $60(48.8)$ \\
\hline & $\geq 3.3$ & $63(51.2)$ \\
\hline \multicolumn{3}{|l|}{ PLR } \\
\hline & $<185$ & $58(47.2)$ \\
\hline & $\geq 185$ & $65(52.8)$ \\
\hline \multicolumn{3}{|l|}{ SII } \\
\hline & $\leq 730$ & $41(33.3)$ \\
\hline 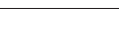 & $>730$ & $82(66.7)$ \\
\hline
\end{tabular}

All analyses were performed with SPSS version 21 (SPSS Inc., Chicago, IL, USA). Data were presented as mean \pm standard deviation or median (1st quartile - 3rd quartile) for continuous variables depending on the normality of distribution. Categorical variables were given as frequency (percentage). Optimum cut-off values were determined by means of receiver operating characteristic (ROC) curve analysis. Survival curves were obtained, and survival rates were determined using the Kaplan-Meier method, and comparisons were made with the log-rank test. Univariate and multivariate analyses using a Cox proportional hazards model were performed to assess potential prognostic factors for OS and PFS. A p-value of 0.05 or lower ( $p$ value $\leq 0.05$ ) was considered statistically significant.

\section{RESULTS}

A total of 123 patients with metastatic adenocarcinoma of 
NSCLC were included retrospectively in the study. The median age of the patients was 60 years (IQR: 55 - 66 years), and mostly male $(n=96,78 \%)$. Most of the patients (89.4\%) were in ECOG PS 0-1. Eighty percent of the patients were current or exsmokers. Otherfeatures of the patients are shown in Tablel.

In the assessment of inflammatory markers, the median SII was 1023.25 (x10 ${ }^{9}$ per 1 L) (IQR: 630.42 - 1742.67), the median NLR was 3.32 (IQR: $2.43-5.20$ ), and the median PLR was 202.25 (IQR: 132.14 - 292.31). In addition, the cut-off value with the highest sensitivity and specificity was determined as 730 (area under the curve (AUC); 0.645, CI 95\%; 0.481-0.808, $\mathrm{p}=0.062$ ), 3.3 (AUC; 0.612, Cl 95\%; 0.470-0.755, $p=0.149$ ), 185 (AUC; $0.561, \mathrm{Cl} 95 \% ; 0.418-0.704, \mathrm{p}=0.434$ ) in ROC curve analysisfor SII, NLR, and PLR respectively. The descriptions were provided SIl-low and SIl-high, NLR-low and NLR- high, PLR-low and PLRhigh due to these cut-off values as shown in Tablel.

In median 14.36 months (IQR: 9.13 - 23.69) of follow-up time, the median PFS was 9 months (CI 95\%: 7.50 - 10.51 months) and the median OS was 15 months ( $\mathrm{Cl} 95 \%$ : 12.40 - 17.63 months). In the univariate analyses; NLR-low group and SII-low group had significantly longer PFS compare to NLR-high and SIl-high groups ( 10 months vs. 8 months, $p=0.018$, and 13 months vs. 8 months, $p<0.001$; respectively, Figure $1-a, b)$. In addition, patients with response to treatment ( $p<0.001$, Figure $1-c)$ had significantly PFS advantage compared to non-responders (Table II). The significant differences were seen between females and males $(p=0.010)$, never smokers and others $(p=0.001$, Figure $1-d)$, responders to treatmentandnon-responders $(p<0.001$, Figure 1-e), and SII-low and SIl-high groups $(p=0.001$, Figure 1-f) according to univariate analyses for overall survival (Table II).

In multivariate analyses, response to treatment and low-SII were independent prognostic factors for PFS (HR: 0.25, $p<0.001$, and HR: 0.47, $p=0.002$, respectively) and OS (HR: 2.09, $p=0.001$, and HR: 2.05, $p=0.001$, respectively, Table II).

The median OS was 18 months (CI 95\%: 15.24 - 20.80 months) and the median PFS was 12 months ( $\mathrm{Cl} 95 \%$ : $10.33-13.70$ months) for the patients received maintenance pemetrexed. As a result of univariate analysis of PFS for the patients received maintenance pemetrexed, the median PFS of SII low group was longer than SII high group (13 months, 95\% Cl:10.64-15.36 months, vs. 10 months, $\% \mathrm{Cl}: 8.41-10.60$ months, $p=0.004)$. The median PFS was 12 months (95\% Cl: 9.80-14.20 months) for NLR low group, and was 10 months ( $95 \% \mathrm{Cl}: 7.25-12.75$ months) for NLR high group ( $p=0.080$ ). There was no statistically significant difference between PLR low and high groups according to median PFS (12 months, 95\% Cl:9.20-14.81 vs. 12 months, 95\% Cl: 10.12-13.90, $p=0.462$ ).
The salient findings of this current work were as follows: Firstly, this study evaluated the prognostic and predictive abilities of SII in patients who had advanced stage adenocarcinoma of lung and underwent first-line pemetrexed cisplatin combination and/or pemetrexed maintenance. Second, the patients in SIIhigh group and NLR-high group had a significantly shorter PFS compared with patients in two low groups. Additionally, SIl-high group had a significant OS disadvantage. The SII, but not NLR and PLR, was an independent prognostic factor for determining survival time in metastatic adenocarcinoma of the patients who underwent pemetrexed therapy. Third, elevated SII after combination therapy was significantly associated with shorter survival after pemetrexed maintenance therapy. Thus, the SII was the best prognostic index strongly correlated with poor survival in comparison with PLR and NLR.

Few studies have compared the prognostic abilities of NLR, PLR, and SII in patients with advanced NSCLC. ${ }^{12}$ Liu and colleagues found that all three inflammatory markers were independently associated with progression-free and overall survival. In contrast to the findings of Liu et al. our results showed that SII was the most powerful predictor of survival times compared with NLR and PLR. Platelet-to-lymphocyte ratio was associated with neither overall nor progression-free survival. On the other hand, Wang et al. found significant PFS differences in terms of NLR, PLR, SII of low and high groups in the univariate analyses. ${ }^{13}$

The neutrophil-to-lymphocyte ratio (NLR) has become a wellestablished and robust inflammatory marker that has prognostic and predictive value in many cancertypes. ${ }^{9,10}$ Afew meta-analyses extended these findings also to non-small cell lung cancer patients. ${ }^{14} A$ number of retrospective studies evaluated pretreatment NLR as a prognostic marker of overall and progression-free survival in patients with advanced-stage NSCLC. While some studies recruited patients on targeted treatments such as nivolumab. ${ }^{2,15}$ others included patients who underwent platinum-based doublet chemotherapy. ${ }^{16}$ Although they reported heterogenous cutoff values, almost all of these studies revealed that higher NLR values were associated with shorter overall and progression-free survival. A few studies also reported that pretreatment NLR value could also predict the response to the treatment. ${ }^{17}$ In current study, the prognostic effect of NLR was evaluated in patients who underwent pemetrexed maintenance. The present results did not confirm the findings of previous studies in terms of the ability of NLR to predict overall survival. However, we found that NLR values $\geq$ 3.3 were significantly associated with shorter PFS but NLR did not appear as an independent predictor of PFS in the Cox regression analysis. This discrepancy with the previous literature might be due to the relatively small sample size and different molecular signature of this study cohort.

\section{DISCUSSION}

Table II: Results of univariate and multivariate analyses for PFS and OS.

\begin{tabular}{|l|l|l|}
\hline & Univariate & Multivariate \\
\hline
\end{tabular}




\begin{tabular}{|c|c|c|c|c|c|c|c|c|c|}
\hline & & $\begin{array}{c}\text { Median PFS (months, 95\% } \\
\mathrm{Cl} \text { ) }\end{array}$ & $p$-value & $\begin{array}{c}\text { Median OS (months, } \\
95 \% \mathrm{Cl} \text { ) }\end{array}$ & $p$-value & $\begin{array}{l}\mathrm{HR}(95 \% \mathrm{Cl}) \\
\text { PFS }\end{array}$ & $\mathrm{p}$-value & HR $(95 \% \mathrm{Cl}), \mathrm{OS}$ & $p$-value \\
\hline & Overall & $9.0(7.5-10.5)$ & & & & - & - & - & - \\
\hline \multicolumn{10}{|c|}{ Age } \\
\hline & $<65$ & $9.0(7.4-10.6)$ & 0.689 & $14.0(11.1-16.9)$ & 0.342 & - & - & - & - \\
\hline & $\geq 65$ & $8.0(5.5-10.5)$ & & $15.0(10.1-19.9)$ & & - & - & - & - \\
\hline \multicolumn{10}{|c|}{ Gender } \\
\hline & Female & $12.0(6.1-17.9)$ & 0.029 & $22.0(14.4-29.6)$ & 0.010 & 1.00 & 0.244 & 1.00 & 0.876 \\
\hline & Male & $9.0(7.7-10.3)$ & & $13.0(10.4-15.6)$ & & $\begin{array}{c}1.33 \\
(0.82-2.16) \\
\end{array}$ & & $\begin{array}{c}0.96 \\
(0.54-1.69) \\
\end{array}$ & \\
\hline \multicolumn{10}{|c|}{ Smoking at diagnosis } \\
\hline & Never & $10.0(5.4-14.6)$ & 0.393 & $22.0(7.6-36.4)$ & 0.001 & - & - & 1.00 & 0.001 \\
\hline & $\begin{array}{l}\text { Current or ex- } \\
\text { smoker }\end{array}$ & $9.0(7.6-10.4$ & & $14.0(11.7-16.3)$ & & - & - & $\begin{array}{c}2.54 \\
(1.45-4.42)\end{array}$ & \\
\hline \multicolumn{10}{|c|}{ Metastatic disease at diagnosis } \\
\hline & No & $12.0(10.0-14.0)$ & 0.122 & $18.0(11.8-24.2)$ & 0.277 & - & - & - & - \\
\hline & Yes & $9.0(7.5-10.5)$ & & $15.0(12.8-17.2)$ & & - & - & - & - \\
\hline \multicolumn{10}{|c|}{ Liver metastasis } \\
\hline & No & $9.0(7.4-10.6)$ & 0.946 & $13.0(10.3-15.7)$ & 0.752 & - & - & - & - \\
\hline & Yes & $9.0(6.4-11.6)$ & & $17.0(11.9-22.1)$ & & - & - & - & - \\
\hline \multicolumn{10}{|c|}{\begin{tabular}{|l|} 
Contralateral lung metastasis \\
\end{tabular}} \\
\hline & No & $9.0(7.4-10.6)$ & 0.740 & $13.0(10.5-15.5)$ & 0.285 & - & - & - & - \\
\hline & Yes & $9.0(6.8-11.2)$ & & $16.0(11.4-20.6)$ & & - & - & - & - \\
\hline \multicolumn{10}{|c|}{ Lymph node metastasis } \\
\hline & No & $12.0(9.0-15.0)$ & 0.181 & $19.0(10.7-27.3)$ & 0.309 & - & - & - & - \\
\hline & Yes & $9.0(7.4-10.6$ & & $14.0(11.9-16.1)$ & & - & - & - & - \\
\hline \multicolumn{10}{|c|}{ Bone metastasis } \\
\hline & No & $8.0(6.4-9.6)$ & 0.628 & $15.0(9.5-20.5)$ & 0.395 & - & - & - & - \\
\hline & Yes & $10.0(7.7-12.3)$ & & $15.0(12.5-17.5)$ & & - & - & - & - \\
\hline \multicolumn{10}{|c|}{ Brain metastasis } \\
\hline & No & $10.0(8.2-11.8)$ & 0.384 & $16.0(13.0-19.0)$ & 0.122 & - & - & - & - \\
\hline & Yes & $8.0(6.4-9.6)$ & & $11.0(7.3-14.7)$ & & - & - & - & - \\
\hline \multicolumn{10}{|c|}{ Radiotherapy } \\
\hline & No & $9.0(7.3-10.7)$ & 0.437 & $15.0(11.5-18.5$ & 0.691 & - & - & - & - \\
\hline & Yes & $9.0(6.6-11.4)$ & & $14.0(9.1-18.9)$ & & - & - & - & - \\
\hline \multicolumn{10}{|c|}{ Response to treatment } \\
\hline & No & $2.0(\mathrm{~N} / \mathrm{A})$ & $<0.001$ & $6.0(0.0-12.3)$ & $<0.001$ & 1.00 & $<0.001$ & 1.00 & 0.002 \\
\hline & Yes & $12.0(10.4-13.7)$ & & $18.0(15.3-20.7)$ & & $\begin{array}{c}0.25 \\
(0.15-0.40) \\
\end{array}$ & & $\begin{array}{c}0.47 \\
(0.29-0.76) \\
\end{array}$ & \\
\hline \multicolumn{10}{|c|}{ NLR } \\
\hline & $<3.3$ & $10.0(8.0-12.0)$ & 0.018 & $15.0(10.0-20.0)$ & 0.079 & 1.00 & 0.481 & 1.00 & 0.376 \\
\hline & $\geq 3.3$ & $8.0(6.4-9.6)$ & & $15.0(13.2-16.8)$ & & $\begin{array}{c}1.17 \\
(0.76-1.81) \\
\end{array}$ & & $\begin{array}{c}0.81 \\
(0.51-1.30) \\
\end{array}$ & \\
\hline \multicolumn{10}{|c|}{ PLR } \\
\hline & $<185$ & $9.0(6.9-11.1)$ & 0.382 & $15.0(11.0-19.0)$ & 0.330 & - & - & - & - \\
\hline & $\geq 185$ & $9.0(7.4-10.6)$ & & $15.0(12.2-17.8)$ & & - & - & - & - \\
\hline \multicolumn{10}{|c|}{ 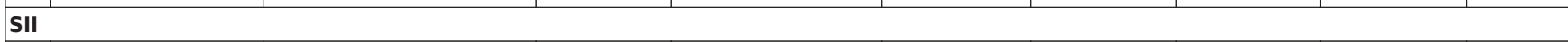 } \\
\hline & $\leq 730$ & $13.0(10.4-15.6)$ & $<0.001$ & $24.0(18.4-29.6)$ & 0.001 & 1.00 & 0.001 & 1.00 & 0.001 \\
\hline & $>730$ & $8.0(6.7-9.3)$ & & $13.0(10.7-15.3)$ & & $\begin{array}{c}2.09 \\
(1.37-3.20)\end{array}$ & & $\begin{array}{c}2.05 \\
(1.33-3.20)\end{array}$ & \\
\hline
\end{tabular}




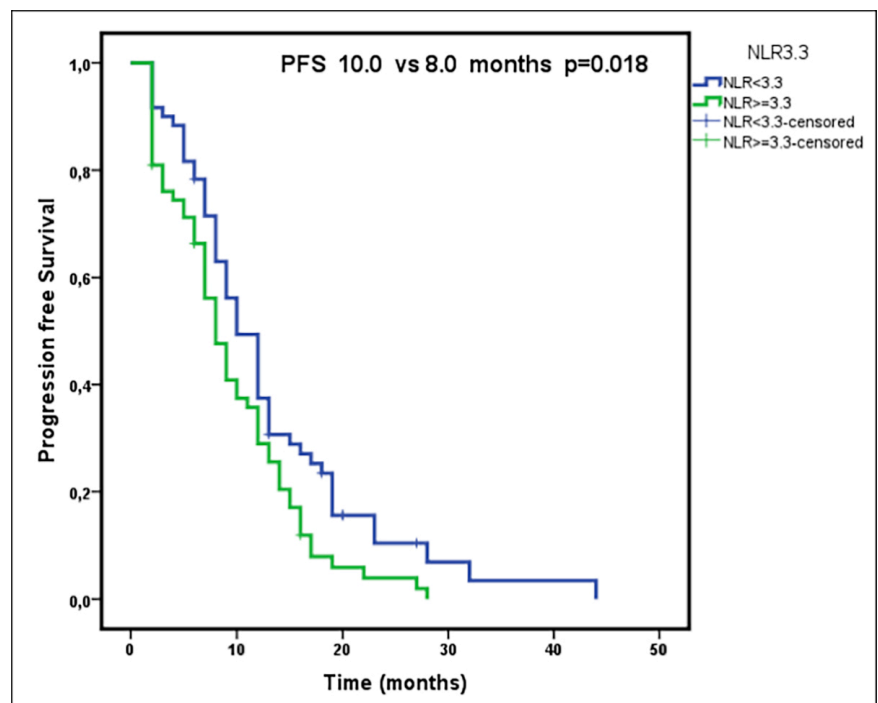

Figure 1a: Progression-free survival in patients NLR $<3.3$ and NLR $\geq 3.3$.

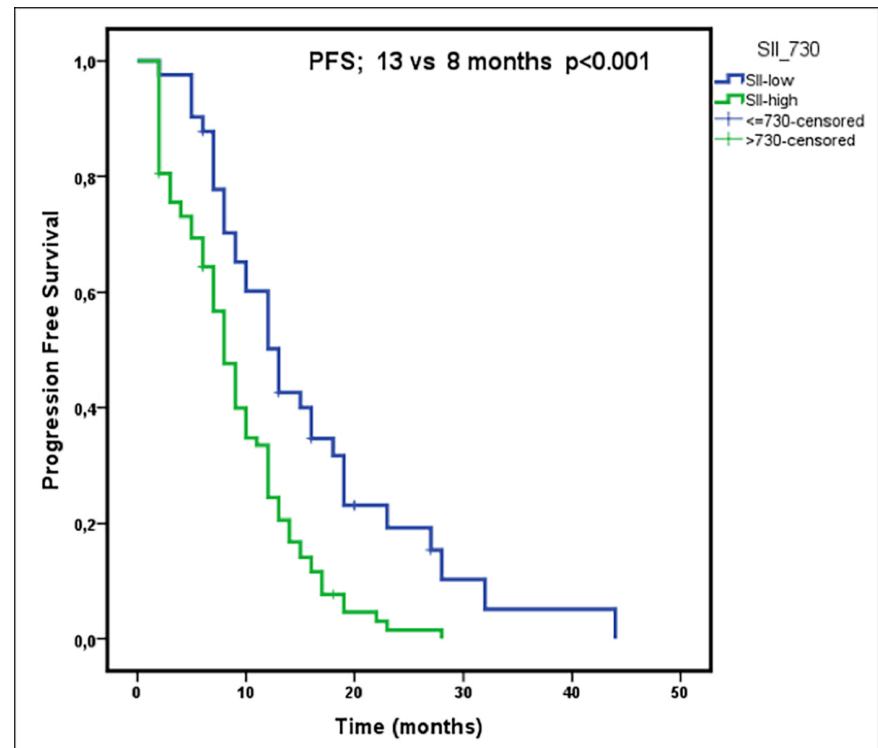

Figure 1b: Progression-free survival in patients SII $\leq 730$ and SII $>730$.

The apparent success of NLR and SII in prediction of survival times and prognosis is related to the biologic roles of neutrophils, lymphocytes, and platelets in cancer patients. Evidence demonstrated that neutrophils might exert immunosuppressive effects that diminish the anti-tumor effects of T-lymphocytes. ${ }^{18}$ Neutrophils can take part in various stages of tumorigenesis, such as tumor growth and metastatic spreading. ${ }^{19}$ In contrast to neutrophils, lymphocytes, especially tumor-infiltrating lymphocytes, have been demonstrated with favorable clinical outcomes. ${ }^{20}$ Platelets can be seen as part of the immune system considering their bridging role between inflammation, thrombosis, and cancer. Platelets support cancer development and progression by a myriad of mechanisms. ${ }^{21}$ Hence, it should not be surprising that when neutrophil, lymphocyte, and platelet numbers are used to produce clinically relevant indices that strongly predict clinical outcomes in patients with cancer. ${ }^{22}$ In this sense, the common feature of many lung cancer studies is that only the power of SII, not PLR, NLR, is shown in multivariate analysis, although all those indices are significantly effective in univariate analysis. ${ }^{22,23}$

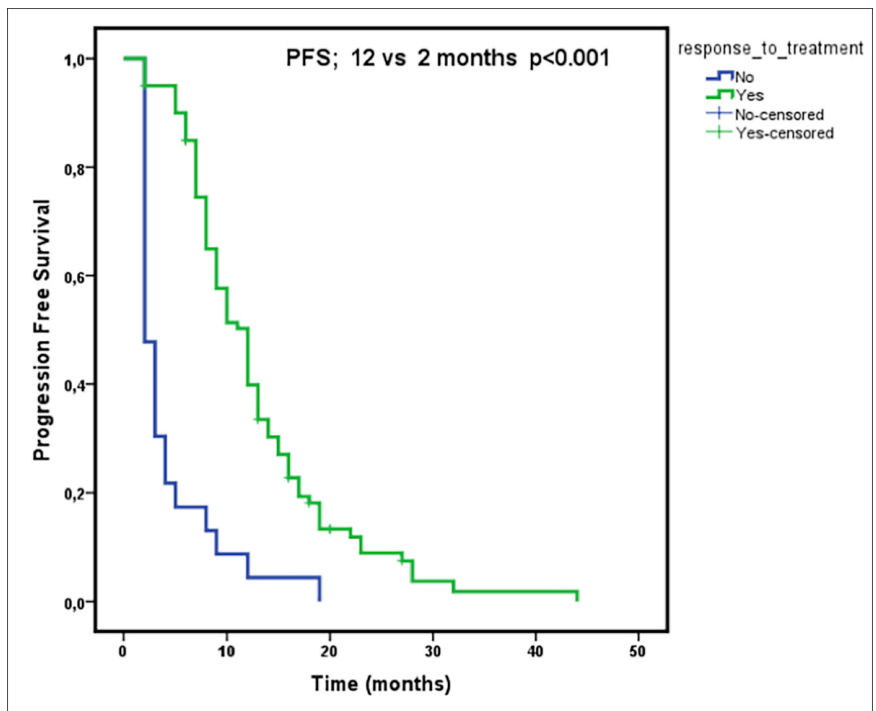

Figure 1c: Progression-free survival in patients; responders and nonresponders to treatment.

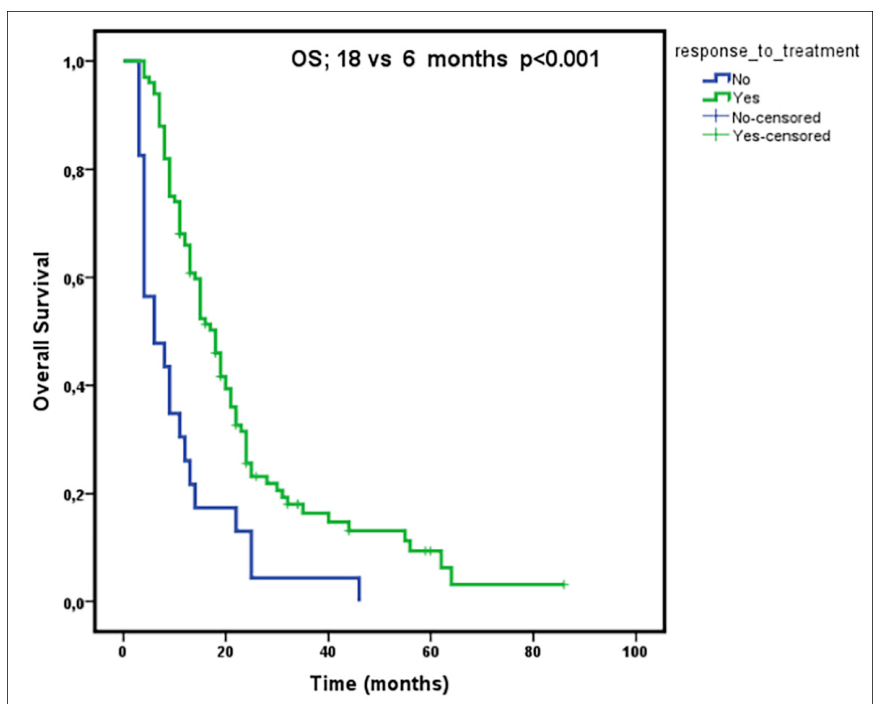

Figure 1d: Overall survival in patients; never smokers vs. current-ex smokers.

SII was recently developed to be able to facilitate the singular abilities of peripheral blood counts to predict clinical outcomes in one equation. ${ }^{24,25}$ A number of studies conducted on patients with advanced NSCLC showed that SII could predict clinical outcomes, including overall and progression-free survival. ${ }^{8,12}$

This study has some limitations. First, a relatively small sample size and retrospective design might have affected the results. Second, it would be better if some biological 
inflammatory markers such as albumin, C-reactive protein, or interleukins are studied, so as to make comparisons.

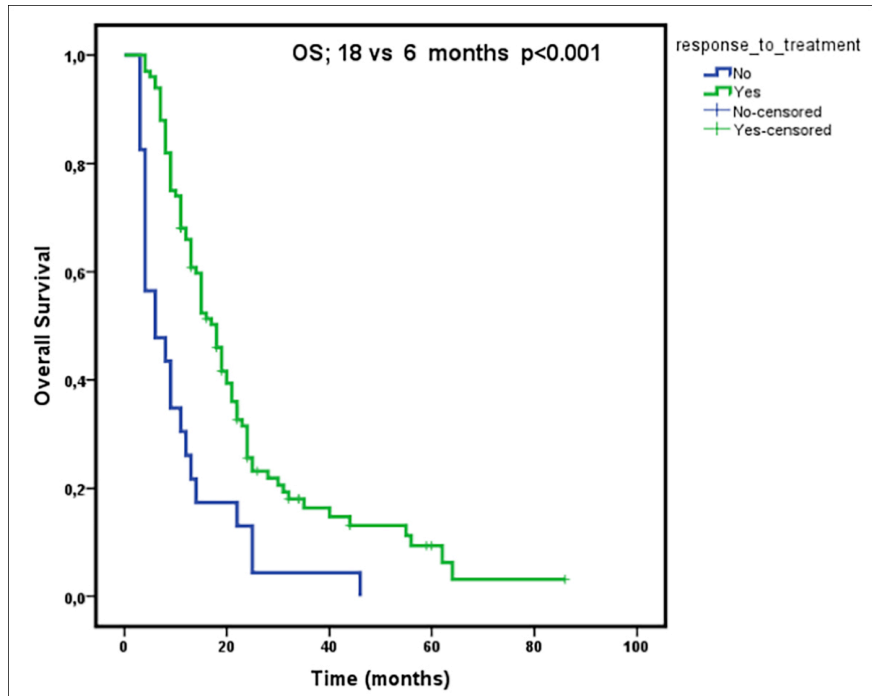

Figure le: Overall survival in patients according to response to treatment.

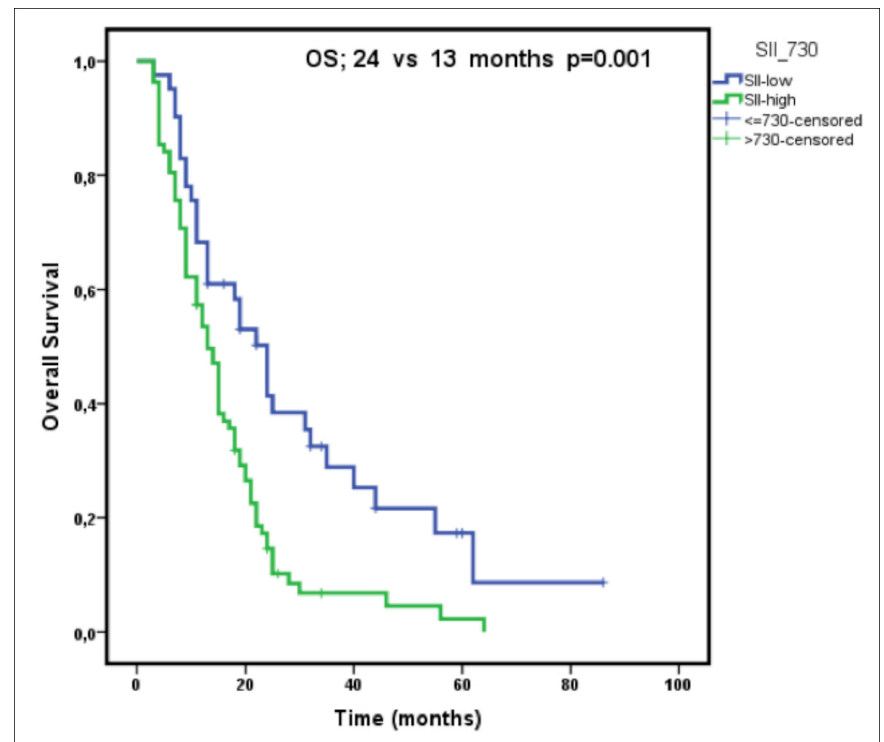

Figure 1f: Overall survival in patients SII $\leq 730$ and SII > 730.

\section{CONCLUSION}

Despite its limitations, this current study contributes valuable novel data to the literature. SII appeared the most powerful of the three studied inflammatory indices, which could independently predict overall and progression-free survival. This study showed the prognostic role of SII, NLR and PLR in advanced stage NSCLC. In addition, SII was detected as independent prognostic factor in terms of PFS and $O S$ in patients who underwent first-line pemetrexed cisplatin combination and/or pemetrexed maintenance.

\section{ETHICAL APPROVAL:}

The study was approved by the local Ethical Committee of the
University of Health Sciences, Dr. A.Y. Ankara Oncology Hospital, before the start of study (TUEK meeting number: 75-30.07.2019).

\section{PATIENTS' CONSENT:}

Informed consents were obtained from all participants or their family, included in the study.

\section{CONFLICT OF INTEREST:}

Authors declared no conflict of interest.

\section{AUTHORS' CONTRIBUTION:}

IB: Acquisition of data, interpretation of data, reviewing the paper, advices, and final approval

FBB: Substantial contributions to conception and design, analysis and interpretation of data, drafting of manuscript, final approval, reviewing the paper.

\section{REFERENCES}

1. Rodriguez-Canales J, Parra-Cuentas E, Wistuba II. Diagnosis and molecular classification of lung cancer. Cancer Treat Res 2016; 170: 25-46. doi: 10.1007/978-3-319-40389-2_2.

2. Reck M, Rabe KF. Precision diagnosis and treatment for advanced non-small-cell lung cancer. N Engl J Med 2017; 377(9):849-61. doi: 10.1056/NEJMra1703413.

3. Hansen $\mathrm{HH}$. Treatment of advanced non-small cell lung cancer. BMJ 2002; 325: 452-3.

4. Hanahan D and Weinberg RA. Hallmarks of cancer: The next generation. Cell 2011; 144(5):646-74. doi: 10.1016/ j.cell.2011.02.013.

5. Grivennikov SI, Greten FR, Karin M. Immunity, inflammation, and cancer. Cell 2010; 140(6):883-99. doi.org/ 10.1016/j.cell.2010.01.025.

6. Diakos Cl, Charles KA, McMillan DC, Clarke SJ. Cancerrelated inflammation and treatment effectiveness. Lancet Oncol 2014; 15(11):e493-503. doi: 10.1016/S14702045(14)70263-3.

7. Wang $Y$, Li Y, Chen $P, X u$ W, Wu Y and Che G. Prognostic value of the pretreatment systemic immune-inflammation index (SII) in patients with non-small cell lung cancer: A meta-analysis. Ann Transl Med 2019; 7(18):433. doi: 10.21037/atm.2019.08.116.

8. Guo D, Zhang J, Jing W, Liu J, Zhu H, Fu L, et al. Prognostic value of systemic immune-inflammation index in patients with advanced non-small-cell lung cancer. Future Oncol 2018; 14(25):2643-50. doi: 10.2217/fon-2018-0285.

9. Eren $T$, Karacin C, Ucar G, Ergun Y, Yazici O, Imamoglu Gl, et al. Correlation between peripheral blood inflammatory indicators and pathologic complete response to neoadjuvant chemotherapy in locally advanced breast cancer patients. Medicine (Baltimore) 2020; 99(22): e20346. doi: 10.1097/MD.0000000000020346.

10. Templeton AJ, McNamara MG, Seruga B, Vera-Badillo FE, Aneja P, Ocana A, et al. Prognostic role of neutrophil-tolymphocyte ratio in solid tumors: A systematic review and meta-analysis. J Natl Cancer Inst 2014; 106(6):dju124. doi: 10.1093/jnci/dju124. 
11. Therasse P, Eisenhauer EA, Verweij J. RECIST revisited: A review of validation studies on tumour assessment. Eur J Cancer 2006; 42(8):1031-9. doi: 10.1016/j.ejca.2006. 01.026

12. Liu J, Li S, Zhang S, Liu Y, Ma L, Zhu J, et al. Systemic immune-inflammation index, neutrophil-to-lymphocyte ratio, platelet-to-lymphocyte ratio can predict clinical outcomes in patients with metastatic non-small-cell lung cancer treated with nivolumab. J Clin Lab Anal 2019; 33(8):e22964. doi: 10.1002/jcla.22964. Epub 2019 Jul 8.

13. Wang D, Guo D, Shi F, Zhu Y, Li A, Kong L, et al. The predictive effect of the systemic immune-inflammation index for patients with small-cell lung cancer. Future Oncol 2019; 15(29):3367-79. doi: 10.2217/fon-2019-0288.

14. Peng B, Wang YH, Liu YM, Ma LX. Prognostic significance of the neutrophil to lymphocyte ratio in patients with nonsmall cell lung cancer: A systemic review and metaanalysis. Int J Clin Exp Med 2015; 8(3):3098-106.

15. Diem S, Schmid S, Krapf M, Flatz L, Born D, Jochum W, et al. Neutrophil-to-lymphocyte ratio (NLR) and Platelet-tolymphocyte ratio (PLR) as prognostic markers in patients with non-small cell lung cancer (NSCLC) treated with nivolumab. Lung Cancer 2017; 111: 176-81. doi: 10.1016/j.lungcan.2017.07.024.

16. Guo D, Li M, Chen D, Jing W, Zhu H, Fu L, et al. Neutrophilto-lymphocyte ratio is superior to platelet-to-lymphocyte ratio as a prognostic predictor in advanced non-small-cell lung cancer treated with first-line platinum-based chemotherapy. Future Oncol 2019; 15(6):625-35. doi: 10.2217/fon-2018-0667.

17. Yao Y, Yuan D, Liu H, Gu X, Song Y. Pretreatment neutrophil to lymphocyte ratio is associated with response to therapy and prognosis of advanced non-small cell lung cancer patients treated with first-line platinum-based chemotherapy. Cancer Immunol Immunother 2013; 62(3):471-9. doi: 10.1007/ s00262-012-1347-9.
18. Petrie HT, Klassen LW, Kay HD. Inhibition of human cytotoxic T lymphocyte activity in vitro by autologous peripheral blood granulocytes. J Immunol 1985; 134(1): 230-4.

19. Coffelt SB, Wellenstein MD, de Visser KE. Neutrophils in cancer: Neutral no more. Nat Rev Cancer 2016; 16(7): 431-46. doi: 10.1038/nrc.2016.52.

20. Al-Shibli KI, Donnem T, Al-Saad S, Persson M, Bremnes RM, Busund LT. Prognostic effect of epithelial and stromal lymphocyte infiltration in non-small cell lung cancer. Clin Cancer Res 2008; 14(16):5220-7. doi: 10.1158/10780432.CCR-08-0133.

21. Franco AT, Corken A, Ware J. Platelets at the interface of thrombosis, inflammation, and cancer. Blood 2015; 126(5): 582-8. doi: 10.1182/blood-2014-08-531582.

22. Hong X, Cui B, Wang M, Yang Z, Wang L, Xu Q. Systemic immune-inflammation index, based on platelet counts and neutrophil-lymphocyte ratio, is useful for predicting prognosis in small cell lung cancer. Tohoku J Exp Med 2015; 236(4):297-304. doi: 10.1620/tjem.236.297.

23. Gao Y, Zhang H, Li Y, Wang D, Ma Y, Chen Q. Preoperative increased systemic immune-inflammation index predicts poor prognosis in patients with operable non-small cell lung cancer. Clin Chim Acta 2018; 484: 272-7. doi: 10.1016/ j.cca.2018.05.059.

24. Hu B, Yang XR, Xu Y, Sun YF, Sun C, Guo W, et al. Systemic immune-inflammation index predicts prognosis of patients after curative resection for hepatocellular carcinoma. Clin Cancer Res 2014; 20(23):6212-22. doi: 10.1158/10780432.CCR-14-0442.

25. Imamoglu GI, Eren T, Baylan B, Karacın C. May high levels of systemic Immune-ınflammation Index and hematologic Inflammation markers suggest a further stage in testicular tumours? Urol Inter 2019; 103(3):303-10. doi: 10.1159/ 000502658. 